\title{
Conducting of Web-Based Workshops for Final Year Medical Students Preparing to Enter the Workforce During the COVID-19 Pandemic
}

\author{
Juliana Yin Li Kan ${ }^{1} \cdot$ Ling Zhu ${ }^{1}$. Nigel Jie Ming Fong ${ }^{2} \cdot$ Xucong Ruan $^{2} \cdot$ Andrew Ming Liang Ong ${ }^{3} \cdot$ Guozhang Lee $^{1}$. \\ Shaikh Abdul Matin Mattar ${ }^{1} \cdot$ Natalie Liling Woong $^{1} \cdot$ Mei Ling Kang ${ }^{1}$
}

Accepted: 13 October 2020 / Published online: 27 October 2020

(C) International Association of Medical Science Educators 2020

\begin{abstract}
Medical students were temporarily removed from direct patient contact activities during the COVID-19 pandemic, shortening the duration of ward-based attachment programs. Web-based workshops were organized to equip final year medical students with necessary skills to start work in a general medicine setting. Topics included case-based scenarios reviewing patients with new complaints, medical documentation, and inter-professional communication. They were conducted using an online video conference platform and utilized polling platforms, small group discussions, and the "Chat" function to promote interactivity. Webbased learning enables delivery of useful contents without compromising interactivity and clinical applicability during the COVID-19 pandemic.
\end{abstract}

Keywords web-based learning $\cdot$ medical student $\cdot$ medical education $\cdot$ COVID-19

\section{Introduction}

Medical education builds upon the foundation of good clinical skills training. Traditionally, medical students acquire various clinical skills through their encounters with patients in hospital. Ward-based learning in an environment with real patients provides learners with not only a variety of case-mix but also the opportunity to develop skills needed for effective communication, safe prescribing, clear record keeping, professionalism, and teamwork.

In 2020, the delivery of ward-based clinical education was greatly hampered by the COVID-19 outbreak. The pandemic has posed multiple challenges to medical education globally. To minimize the risk of exposure of medical students to COVID-19 and to avoid medical students being potential vectors for transmission, medical students were removed from

Juliana Yin Li Kan

juliana.kan.y.1@singhealth.com.sg

1 Department of Internal Medicine, Singapore General Hospital, Singapore, Singapore

2 Ministry of Health Holdings Pte Ltd, Singapore, Singapore

3 Department of Gastroenterology and Hepatology, Singapore General Hospital, Singapore, Singapore direct patient contact activities in many institutions [1,2]. Social distancing measures preclude students from attending traditional education settings such as lectures.

The Duke-NUS Medical School is a graduate-entry medical school in Singapore. All final year medical students traditionally embark on an 8-week Student-in-Practice (SIP) program in preparation for starting work as a Post Graduate Year One (PGY1) doctor. It takes place after the Clinical Performance Examination at the end of year 4, prior to graduation. During this period, students are embedded into a medical team for 4 weeks, and a surgical team for 4 weeks. Students shadow junior doctors on the team as they learn to deliver patient care. The Department of Internal Medicine, Singapore General Hospital (SGH), is one of the hosting departments of SIP students. It is the largest department of SGH, a large acute tertiary hospital in Singapore. In addition to having a close partnership with the Duke-NUS Medical School, the department provides postgraduate training to hundreds of PGY1 doctors each year.

On 7th February 2020, in response to the COVID-19 situation, Singapore raised the risk assessment level of the fourtier Disease Outbreak Response System Condition (DORSCON) to Orange, the second highest level. Singapore's Ministry of Health (MOH) subsequently issued directives to suspend clinical activities for all medical students nationwide, disallowing them from entering hospital grounds. 
This occurred 7 weeks before the scheduled commencement of the SIP program on 30th March 2020. As these medical students were nearing graduation and entry to the healthcare workforce in June, it was imperative that they received the training and preparation necessary to function as competent PGY1s. The challenge was to find an appropriate online substitute for ward-based learning.

In this paper, we describe a series of web-based workshops organized for final year medical students, to equip them with the necessary clinical and administrative skills to start work as a competent doctor in a general medicine setting during the COVID-19 pandemic.

\section{Methods}

The workshops consisted of five 2-h sessions delivered to 56 final year medical students from the Duke-NUS Medical School over a period of 2 weeks. The faculty consists of clinicians of the Department of Internal Medicine, Singapore General Hospital, and residents from the Internal Medicine Residency Program, SingHealth. All tutors have experience in undergraduate medical education and PGY1 training. All teaching materials were reviewed and approved by at least one tutor with accreditation by the Specialists Accreditation Board, Singapore. Simulated patient data was used for case illustrations and exercises to maintain confidentiality of real patient data. An optional, non-anonymized pre-workshop and postworkshop survey was conducted, asking students to rate their confidence levels in performing tasks expected of a competent PGY1. Written feedback was also gathered after every session.

\section{Content Development}

During the planning of the content of these workshops, we aimed to achieve the following objectives:

(a) Equip students with skills needed to practice as a PGY1 doctor in an internal medicine ward

(b) Map deliverables according to recommendations from the National Medical Undergraduate Curriculum Committee (NMUCC) which are aligned to the Accreditation Council for Graduate Medical Education (International) (ACGME-I) core competencies [3], and the National Assessment Committee for Post Graduate Year 1 (NAC-PGY1) [4]

(c) Maximize interactivity between the faculty and learners

(d) Strict adherence to infection control protocols and social distancing measures

Emphasis was placed on conditions and scenarios commonly seen in medical wards. In addition, faculty members of different levels of seniority (according to post graduate years) met up to discuss and identify common areas of deficiencies in PGY1 doctors rotating through the Department of Medicine, Singapore General Hospital. Other learning activities organized by the school, such as simulation sessions for hands-on bedside procedures, workshops for communication skills, ethics and professionalism and patient safety were reviewed and not repeated in our series of workshops (henceforth referred to as web-based medicine SIP workshops).

A total of four topics were shortlisted to cover the learning objectives of web-based medicine SIP workshops (Table 1): called to see patient (CTSP) scenarios, medical documentation, writing a discharge summary, and making a referral.

\section{Delivery of Workshops}

The workshops were conducted through video conferencing, utilizing Zoom (Zoom Video Communications Inc., 2020) as the online web platform. Students participated from home. Tutors were provided internet-enabled laptops and were given the option of conducting the workshop from their own office or from a designated room in the school. Tutors were given guides on how to operate Zoom, and the school administrative team conducted dry runs as necessary for tutors to familiarize themselves with Zoom functions. Entry to Zoom meetings was password protected, and students were instructed to turn on their web cameras at all times for proof of identity and attendance.

To promote interactivity, many workshops utilized Poll Everywhere (Poll Everywhere Inc, CA, USA, 2020), an online dynamic polling platform, to engage students in answering questions. Students could ask questions via speaking through their audio devices or the "Chat" function. A supervising senior doctor was present in every session. As some workshops had multiple tutors, the supervising tutor could answer questions and clarify doubts in the chat group promptly without interrupting the presenter. Many sessions used breakout rooms to facilitate small-group discussions.

\section{Called to See Patient (CTSP) Scenarios}

"CTSP" is a phrase prevalent in our local medical community, referring to a doctor's approach to reviewing a patient with a new complaint. This workshop was made up of two sessions and covered practical tips on common CTSP scenarios. This included how to triage patient reviews, how to perform a postprocedure review, the approach to abnormal vital signs and delirium, and electrocardiogram interpretation. Teaching was conducted using a "hot seat" format, where students were asked how they would respond in case scenarios, and how they would communicate information and instructions to other healthcare professionals. Choice of this educational method was intentional to simulate the decision-making process and develop clinical reasoning. Commonly made mistakes were highlighted to learners. As researchers predict COVID-19 
Table 1 Outline of competencies achieved by each web-based medical SIP workshop

\begin{tabular}{|c|c|c|c|c|c|}
\hline & \multirow[t]{2}{*}{ Professional activity/core competency } & \multicolumn{4}{|c|}{ Workshop } \\
\hline & & CTSP $^{\mathrm{a}}$ & Documentation & Referral & $\begin{array}{l}\text { Discharge } \\
\text { summary }\end{array}$ \\
\hline \multirow[t]{7}{*}{$\begin{array}{l}\text { Professional activities outlined by } \\
\text { the National Assessment } \\
\text { Committee for PGY1 }\end{array}$} & $\begin{array}{l}\text { Manage patients by obtaining a detailed history, performing } \\
\text { appropriate physical examination, requesting and } \\
\text { follow-through relevant diagnostic evaluation and therapeutic } \\
\text { interventions }\end{array}$ & $\checkmark$ & & & \\
\hline & Manage transitions of care (within and between teams) & & $\checkmark$ & $\checkmark$ & $\checkmark$ \\
\hline & Able to perform bedside procedures & & & & \\
\hline & $\begin{array}{l}\text { Resuscitate, stabilize, and care for unstable or critically ill } \\
\text { patients }\end{array}$ & $\checkmark$ & & & \\
\hline & $\begin{array}{l}\text { Provide peri-procedural and post-procedural/surgical assessment } \\
\text { and care }\end{array}$ & $\checkmark$ & & & \\
\hline & $\begin{array}{l}\text { Able to educate, update, and interact with patient and family } \\
\text { members and facilitate family conferences }\end{array}$ & & & & \\
\hline & $\begin{array}{l}\text { Improve the quality of healthcare at both individual and systems } \\
\text { levels }\end{array}$ & & & & \\
\hline \multirow[t]{6}{*}{ ACGME-I ${ }^{\mathrm{b}}$ core competencies } & Patient care & $\checkmark$ & $\checkmark$ & $\checkmark$ & $\checkmark$ \\
\hline & Medical knowledge & $\checkmark$ & $\checkmark$ & $\checkmark$ & $\checkmark$ \\
\hline & Practice-based learning and improvement & $\checkmark$ & & & \\
\hline & Interpersonal and communication skills & & $\checkmark$ & $\checkmark$ & $\checkmark$ \\
\hline & Professionalism & & & & \\
\hline & System-based practice & & $\checkmark$ & $\checkmark$ & $\checkmark$ \\
\hline
\end{tabular}

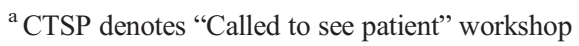

b ACGME-I denotes "Accreditation Council for Graduate Medical Education (International)"

transmissions to persist in the months ahead [5], we included a case scenario involving the management of a patient with suspected COVID-19. There was deliberate attention given to COVID-19-related precautions and workflows, familiarization with systems-based practices, and integration of theoretical and clinical knowledge. It taught students how to suspect and diagnose a case of COVID-19, including instructions on donning appropriate personal protective equipment and the workflow on isolating the patient.

\section{Medical Documentation and Writing a Discharge Summary}

In Singapore General Hospital, all patient-related activities and processes are documented in the hospital's electronic medical record (EMR) system by healthcare professionals. Two workshops taught students how to document inpatient daily ward round notes, and how to summarize a patient's admission in a discharge summary. Suggested frameworks and templates were provided to give students guidance on the essential components of each type of document. Students were given a preworkshop assignment and submitted a discharge summary based on an anonymized clinical scenario. The case was deliberately written up with in-depth details to simulate a complex patient. This exercise challenged them to navigate the system and labyrinth of information. Anonymized positive and negative examples submitted by students from the preworkshop assignments were presented to illustrate teaching points. Students were given the opportunity to resubmit their discharge summary for review after the workshop.

\section{Making a Referral}

This workshop was designed to teach students how to consult a specialist, including how to make a good referral with a clear clinical question, interpret the reply, and update a healthcare colleague on a patient's progress. To facilitate transition of care in between healthcare providers, this workshop also provided guidance and instruction on how to write a good handover memo using the Situation, Background, Assessment, Recommendation (SBAR) technique.

\section{Events After the Medicine SIP Workshops}

In late March 2020, the Ministry of Health of Singapore approved gradual resumption of clinical training for medical students. Following the completion of the 2-week web-based medicine SIP workshops, the class commenced their wardbased SIP program on 13th April 2020. Effectively, the workshops functioned as front-loading foundational tutorials prior 
to ward-based rotations. Graduation took place in May and the class commenced their PGY1 training on 22nd June 2020.

\section{Discussion}

COVID-19 has changed the landscape of medical education, with a surge in reliance on the use of online learning due to limitations imposed on face-to-face teachings and patient exposure. It has provided a strong impetus to evaluate what knowledge and clinical skills traditionally acquired through ward-based learning can be taught effectively online [6]. One strategy that has been adopted is the introduction of "virtual clerkships" with online modules comprising of lectures on disease entities with paired assessment tools to compensate for shortened in-person learning time [7].

The learning objectives of a graduating medical student due to enter the workforce, however, are unique. Traditionally, a portion of the SIP program involves direct patient and family encounters, including history taking, physical examination, hands-on procedures, and patient and family communication. A significant amount of time is also spent in a non-patientfronting setting. This includes analyzing results and formulating plans, documenting patient encounters, inter-professional communication with healthcare workers, and preparing documents for transition of care. Interpersonal skills are foundational for medical practitioners. The ability to communicate effectively with physicians, other healthcare professionals, and health-related agencies is closely linked with issues of patient safety and medical error [8]. In addition, maintenance of comprehensive, timely, and legible medical records aids clinical care planning among different caregivers in a complex healthcare system [9]. Attaining competency in performing these tasks does not necessitate patient interaction, and hence was the focus of the web-based medicine SIP workshops.

We employed several strategies to maximize the learning value of the workshops. For the CTSP workshops, we invited young internal medicine residents to helm the sessions. This allowed integration of theory with practical tips. Through both the tutors' own experience and the years of experience in PGY1 training, we were able to collectively compile a list of commonly encountered scenarios and commonly made mistakes. Conducting the teaching to the entire class simultaneously enabled a consistent experiential learning experience across all learners, thereby overcoming the limitations of the less-structured on-the-job learning. Previously, depending on the patients encountered, enthusiasm of the learner, and eagerness of the teacher, the learning experience is opportunistic and varied. The lack of structure and standardized teaching also results in differences in practices and proficiency in performing tasks. This initiative ensured that all medical students would have an accurate understanding of the essentials to minimize the development of erroneous and misguided work practices. To facilitate hands-on practice for medical documentation, students were given a pre-workshop assignment. With this, students could attempt to write a sample discharge summary and have it evaluated in a safe environment. Open, non-judgmental discussions on the strengths and weaknesses of each excerpt allowed students to learn from their peers. The opportunity for students to rewrite the discharge summary further reinforced their learning.

Whilst learning needs of SIP students are clear, preparing a web-based curriculum to deliver relevant contents for these students was challenging [10]. It required dedicated faculty and residents who invested time to prepare content and familiarize themselves with online video conferencing and polling platforms. The execution of workshops was involved many hours of work by the physicians and the supporting administrative team. In particular, the discharge summary workshop involved the tutors reviewing more than 50 copies of student submissions, which was a labor-intensive process. Due to the rapidly evolving nature of the COVID-19 situation in the early phase of the pandemic, we organized the SIP workshops within a limited timeframe. We placed strong emphasis on open communication and feedback from students, and therefore did not mandate the anonymity of feedback collected. As a result, although students responded enthusiastically to our workshops and feedback from tutors was positive, we are unable to utilize the data for the purpose of publication.

Through the organization of this series of workshops, we have uncovered a number of limitations of this method of medical education. In addition to the lack of interactions with real patients, it is evident that certain clinical skills, such as hands-on procedures and communication with patients and families, cannot be taught comprehensively via an online platform. Furthermore, prolonged web-based learning may limit the "sense of belonging" of students. A critically important part of medical education takes place in communities of practice, where individuals move from legitimate peripheral to full participation in the healthcare team [11]. This highlights the social nature of learning, helps with identity formation, and develops camaraderie which is crucial to the morale of healthcare teams.

Since the workshop that took place in April 2020, students have gradually been allowed to return to clinical areas, with strict infection control and social distancing measures in place. As we are adapting to the "new norm" in this COVID-19 pandemic, we hope that the web-based medicine SIP workshops would complement traditional clinical training and augment the subsequent clinical learning experience. Moving forward, plans are underway in our institution to incorporate these web-based teachings into curriculum, so that future batches of students can continue to benefit from the material. To encourage sustainable delivery of these workshops, a flipped classroom approach is proposed. Pre-recorded voiceannotated presentations are to be reviewed by students in their own time, followed by an interactive session with tutors to 
answer their questions, review assignments, and have smallgroup discussions. This will circumvent logistical issues for students posted to different institutions for their hospital attachments. To refine the material and delivery of content, we will integrate education research methodologies when collecting feedback for future batches of SIP students. Lastly, assessments documenting improved competency of learners in various skill and knowledge domains are necessary to objectively evaluate the success of the program.

\section{Conclusion}

The COVID-19 pandemic has posed unprecedented challenges to medical education. However, technology has enabled the delivery of many useful contents without necessarily compromising interactivity and clinical applicability. Whilst the importance of hands-on clinical experience cannot be over-emphasized, web-based learning has proven to be a valuable tool that will break through the traditional geographic barriers in medical education.

\section{References}

1. Guidance on medical students' participation in direct patient contact activities. Association of American Medical Colleges (14 April 2020).
2. Advice from Medical Schools Council to UK Medical Schools on actions surrounding Covid-19. Medical Schools Council (13 March 2020).

3. Outcomes and Standards for Undergraduate Medical Education in Singapore. Recommendations of the National Medical Undergraduate Curriculum Committee (2014).

4. Implementation Note for the National PGY1 Training and Assessment Framework. National Assessment Committee for PGY1 (June 2014).

5. Institute for Health Metrics and Evaluation. Available at: http:// www.healthdata.org/covid/updates. Accessed 11 Aug 2020.

6. Sandars J, Correia R, Dankbaar M, de Jong P, Goh PS, Hege I, Masters K, Oh SY, Patel R, Premkumar K, Webb A. Twelve tips for rapidly migrating to online learning during the COVID-19 pandemic. MedEdPublish. 2020 Apr;29:3068.

7. Calhoun KE, Yale LA, Whipple ME, Allen SM, Wood DE, Tatum RP. The impact of COVID-19 on medical student surgical education: implementing extreme pandemic response measures in a widely distributed surgical clerkship experience. Am J Surg. 2020;220: 44-7.

8. Varpio L, Hall P, Lingard L, Schryer CF. Interprofessional communication and medical error: a reframing of research questions and approaches. Acad Med. 2008;83(10):S76-81.

9. Benson BJ. Domain of competence: interpersonal and communication skills. Acad Pediatr. 2014;14(2 Suppl):S55-65.

10. O'Doherty D, Dromey M, Lougheed J, Hannigan A, Last J, McGrath D. Barriers and solutions to online learning in medical education - an integrative review. BMC Med Educ. 2018;18(1): 130.

11. Cruess RL, Cruess SR, Steinert Y. Medicine as a community of practice: implications for medical education. Acad Med. 2018;93(2):185-91.

Publisher's Note Springer Nature remains neutral with regard to jurisdictional claims in published maps and institutional affiliations. 